\title{
THE RESTRICTED PROBLEM OF THREE BODIES
}

\author{
BY \\ MONROE H. MARTIN
}

The difficulty of the problem of three bodies led Jacobi( $\left.{ }^{1}\right)$ to introduce a simplifying assumption, designed to make the problem more amenable to mathematical attack, but such that the problem retains its astronomical significance. In the restricted problem of three bodies Jacobi postulated that two masses known as finite masses revolve perpetually in concentric circles about their common center of mass in accordance with the laws of the two body problem and required the motion of a third mass termed the infinitesimal mass under the assumption that it is attracted by the two finite masses according to the Newtonian law of gravitation.

In this paper the scope of the problem is enlarged by permitting the two finite masses to move in accordance with an arbitrarily chosen solution of the two body problem. One is immediately led to three types of restricted problems according as one finite mass moves in an ellipse $\left({ }^{2}\right)$, parabola or hyperbola about the other as focus. As might be expected, considerable simplification occurs when the conic section degenerates into a line segment.

A restricted problem of three bodies may always be reduced to a quasiLagrangian system $\left({ }^{3}\right)$

$$
\frac{d}{d t} \frac{\partial L}{\partial \dot{q}_{r}}-\frac{\partial L}{\partial q_{r}}+k \frac{\partial L}{\partial \dot{q}_{r}}=0, \quad L=L\left(q_{r}, \dot{q}_{s}, t\right), \quad k=k(t),
$$

by the introduction of suitable variables. Such systems reduce to Lagrangian systems upon introduction of a Lagrangian function $\bar{L}=e^{l} L$, where $l=\int k d t$. Parts I, II, III of the paper are devoted to a study of these systems, partly with a view of their applications to the restricted problem of three bodies

Presented to the Society in two parts, the first under the title Restricted problems in three bodies on December 29, 1938 and the second under the title Quasi-Lagrangian systems on April 26, 1940; received by the editors June 27, 1941, and, in revised form, March 27, 1942.

(1) R. Marcolongo, Il problema dei tre corpi, Milan, 1919, p. 97, ascribes the problem to Jacobi. Recently the following papers, among others, have appeared on the problem: G. D. Birkhoff, Sur le probleme restreint des trois corps, Annali della R. Scuola Normale Superiore di Pisa, (2), vol. 4 (1935), pp. 1-40 (first memoir) and (2), vol. 5 (1936), pp. 1-42 (second memoir); A. Wintner, Beweis des E. Strömgrenschen dynamischen Abschlussprincips der periodischen Bahngruppen im restringierten Dreikörperproblem, Mathematische Zeitschrift, vol. 34 (1931), pp. 321349, where further references are given. See also A. Wintner, The Analytical Foundations of Celestial Mechanics, Princeton, 1941.

(2) The restricted problem of elliptic type has been investigated by F. R. Moulton, Periodic Orbits, Carnegie Institute of Washington Publication, 1920, pp. 217-284.

(3) Special cases of these systems have been considered by Elliott. See P. Appell Traité de Mécanique Rationelle, Paris, 1902, vol. 1, pp. 582-583, where further references are given. 
and partly because of their intrinsic interest as examples of non-conservative systems. Among other things, their "limiting motions" are studied; in particular, conditions sufficient to insure that a motion tends toward equilibrium are obtained. For quasi-conservative ( $L_{t} \equiv 0, k=$ const.) systems it is possible to obtain a generalization of the energy integral and of the principle of least action, the latter involving a Mayer calculus of variations problem. Parts IV, V, VI deal with the restricted problem of three bodies. In the main they are concerned with the behavior of the infinitesimal mass as the two finite masses recede to infinity or approach (or leave) a collision along a degenerate conic section.

\section{QUASI-LAGRANGIAN SYSTEMS}

1. General principles. A dynamical system with $n$ degrees of freedom and equations of motion( $\left.{ }^{4}\right)$

$$
\frac{d}{d t} \frac{\partial L}{\partial \dot{q}_{r}}-\frac{\partial L}{\partial q_{r}}+k \frac{\partial L}{\partial \dot{q}_{r}}=0, \quad L=\left(a_{r s} \dot{q}_{r} \dot{q}_{s} / 2\right)+b_{r} \dot{q}_{r}+c, \quad k=k(t),
$$

is termed a quasi-Lagrangian system. If $k$ is a positive constant, the quasiLagrangian system becomes a dissipative system for which Rayleigh's dissipation function $\left(^{5}\right)$ is proportional to the Lagrangian function. It is readily verified that the equations of motion may be given the variational form

$$
\delta \int_{t_{0}}^{t_{1}} e^{l} L d t=0, \quad l=\int k d t
$$

there being no variation in the time $t$, nor in the end points of the varied curves. Subjected to Legendre's transformation they take the canonical form( $\left(^{6}\right)$

$$
\dot{q}_{r}=H_{p_{r}}, \quad \dot{p}_{r}=-H_{q_{r}}-k p_{r}, \quad H=\left(a^{r s} 2 /\right)\left(p_{r}-b_{r}\right)\left(p_{s}-b_{s}\right)-c,
$$

the integration of which is equivalent to the determination of a complete solution of the partial differential equation

$$
S_{t}+k S+H\left(t, q_{r}, S_{q_{s}}\right)=0 .
$$

This partial differential equation accordingly plays the role of the HamiltonJacobi partial differential equation.

In place of Liouville's theorem which likens the flow in the phase space

(4) All functions are assumed analytic in their arguments. The dot denotes differentiation with respect to the time $t$. The matrix $\left\|a_{r s}\right\|$ is assumed to be positive definite and the repeated indices denote summation from 1 to $n$.

(5) For the theory of Rayleigh's dissipation functions see E. T. Whittacker, A Treatise on the Analytical Dynamics of Particles and Rigid Bodies, Cambridge, 1927, pp. 230-231.

(6) The reciprocal matrix of $\left\|a_{r s}\right\|$ is denoted by $\left\|a^{r s}\right\|$. 
of a Lagrangian system to that of an incompressible fluid, the flow in the phase space of a quasi-Lagrangian system obeys the law $V e^{n l}=$ const., where $V$ is the $2 n$-dimensional volume of a portion of the phase space at time $t$. To prove this, one notes that the condition $\left({ }^{7}\right)$ for $\int M d V$ to be an integral invariant of (3) yields $M e^{-n l}=$ const.

The rate at which the energy $H$ is changing along a motion of (3) is expressed by

$$
d H / d t=-k p_{r} H_{p_{r}}+H_{t}
$$

and the system is termed acquisitive or dissipative in a certain time interval according as $d H / d t \gtrless 0$ holds in this time interval for every motion of the system. The two forms of $H$ important for the restricted problem of three bodies turn out to be

$$
\begin{aligned}
& H=\left(a^{r_{s}} / 2\right)\left(p_{r}-e^{-l} b_{r}\right)\left(p_{s}-e^{-l} b_{s}\right)-c, \\
& H=\left(a^{r_{s}} / 2\right)\left(p_{r}-e^{-l} b_{r}\right)\left(p_{s}-e^{-l} b_{s}\right)-e^{-l} c,
\end{aligned}
$$

where $a^{r s}, b_{r}, c$ are functions of $q_{r}$ only. One finds

$$
\begin{aligned}
d H / d t & =-k a^{r s}\left(p_{r}-e^{-l} b_{r}\right)\left(p_{s}-e^{-l} b_{s}\right), \\
d\left(e^{l} H\right) / d t & =-(k / 2) e^{l} a^{r s}\left(p_{r}-e^{-l} b_{r}\right)\left(p_{s}-e^{-l} b_{s}\right),
\end{aligned}
$$

holding, respectively, so that in both cases the system is acquisitive or dissipative according as $k \lessgtr 0$.

2. Limiting motions. A motion $\bar{M}$ defined( $\left.{ }^{8}\right)$ for $a<t<b$ is an $\omega$-limiting motion of a motion $M$ defined for $t>t_{0}$ if, given any subinterval $a_{0} \leqq t \leqq b_{0}$ of $a<t<b$, an arbitrarily fixed, small positive number $\delta$, and an arbitrarily fixed, large positive number $T$, there exists a $\tau>T$ such that the point $P(t)$ on $M$ is at a distance less than $\delta$ from the point $\bar{P}(t-\tau)$ on $\bar{M}$ for $a_{0} \leqq t-\tau \leqq b_{0}$. An $\omega$-limiting point of $M$ is a point of accumulation of a sequence of points $P\left(t_{i}\right)$ on $M$ for which $t_{i} \rightarrow+\infty . \alpha$-limiting motions and $\alpha$-limiting points are defined similarly for $t \rightarrow-\infty$. Assuming that the second members in (3) are regular analytic in $q_{r}, p_{s}, t$ throughout the phase space with the exception of the points of a set $S$ at which singularities occur for $t>t_{0}$, a motion defined for $t>t_{0}$ is positively stable if it remains in a bounded portion of the phase space and does not come arbitrarily close to $S$, otherwise $M$ is positively instable. Negatively stable (instable) motions are defined similarly and a motion both positively and negatively stable is termed stable.

If $H, H_{q_{r}}, H_{p_{\text {s }}}$ tend towards limiting functions $\bar{H}, \bar{H}_{q_{r}}, \bar{H}_{p_{\varepsilon}}$ uniformly in any bounded, closed subregion of the phase space not containing points of $S$ while $k$ tends toward a finite limit $\bar{k}$ as $t \rightarrow+\infty$, the $\omega$-limiting motions of a

(7) See E. T. Whittacker, op. cit., pp. 283-284.

(8) The possibilities $a=-\infty, b=+\infty$ are not to be excluded. 
motion $M$ of $(3)$ are $\left(^{9}\right)$ motions of the limiting system

$$
\dot{q}_{r}=\bar{H}_{p_{r}}, \quad \dot{p}_{r}=-\bar{H}_{q_{r}}-\bar{k} p_{r} .
$$

In case $M$ is positively stable its $\omega$-limiting motions comprise a set of stable motions approached uniformly by $M$ as $t \rightarrow+\infty$.

THEOREM 1. If the Hamiltonian function $H$ has either form in (5) and if $k$ tends toward a finite positive limit $\bar{k}$ as $t \rightarrow+\infty$ the $\omega$-limiting motions of a positively stable motion $M$ of (3) are equilibrium motions $\left({ }^{9}\right)$ of the system (6) with $\bar{H}=\left(a^{r s} p_{r} p_{s} / 2\right)-c$, or $\bar{H}=a^{r s} p_{r} p_{s} / 2$.

Along $M$ the energy $H$ is eventually a monotone decreasing function of $t$ and will accordingly approach a finite limit $\bar{h}$, since $M$ is positively stable. Along an $\omega$-limiting motion $\bar{M}$ of $M$ we have $\bar{H}=\bar{h}$. To see this let $\bar{P}$ be any point of $\bar{M}$ and $P$ be the point on $M$ at time $t$. Clearly

$$
|\bar{H}(\bar{P})-\bar{h}| \leqq|\bar{H}(\bar{P})-\bar{H}(P)|+|\bar{H}(P)-H(P)|+|H(P)-\bar{h}| .
$$

Let $\epsilon$ be an arbitrarily small positive number. In view of the uniform convergence of $H$ towards $\bar{H}$ we may select $t_{1}$ such that $|\bar{H}(P)-H(P)|<\epsilon$ for all $t>t_{1}$. Since $H(P)$ tends to $\bar{h}$ as a limit, there exists a $t_{2}$ for which $|H(P)-\bar{h}|<\epsilon$ for all $t>t_{2}$. Finally $\bar{H}(P)$ is a continuous function of $P$ and $\bar{P}$ is an $\omega$-limiting point of $M$. Hence there exists a value of $t$ greater than either $t_{1}$ or $t_{2}$ for which $|\bar{H}(\bar{P})-\bar{H}(P)|<\epsilon$. It follows that $|\bar{H}(\bar{P})-\bar{h}|$ is arbitrarily small and therefore equals zero.

Along $\bar{M}$ we have $d \bar{H} / d t=-\bar{k} a^{r s} p_{r} p_{s}=0$ and therefore, since $\left\|a^{r s}\right\|$ is positive definite, the $p_{r}$ are zero on $\bar{M}$. Hence $\bar{M}$ is an equilibrium motion.

It is obvious that these systems possess no periodic motions.

\section{QUASI-CONSERVATIVE SYSTEMS}

1. General principles. A quasi-Lagrangian system is termed quasi-conservative if $L_{t} \equiv 0$ and $k$ is a constant other than zero. For such systems there exists a generalization of the energy integral

THEOREM 2. If $S$ be defined along a motion $M$ by

$$
e^{k t} S=e^{k t} S_{0}+\int_{t_{0}}^{t} e^{k t}\left(p_{r} H_{p_{r}}-H\right) d t,
$$

the quantity $(H+k S) e^{k t}$ retains a constant value along $M$.

Placing $z=S, p=S_{t}, p_{r}=S_{q_{r}}$ in (4), the differential equations of the char-

( $\left.{ }^{9}\right)$ For the treatment of steady flows, see G. D. Birkhoff, Quelques théorèmes sur le mouvement des systèmes dynamiques, Bulletin de la Société Mathématique de France, vol. 40 (1912), pp. 305-323. For a treatment of dissipative systems not involving the time explicitly, see his book Dynamical Systems, American Mathematical Society Colloquium Publications, vol. 9, 1927, pp. 31-32. 
acteristic strips of (4) are

$$
t^{\prime}=1, \quad q_{r}^{\prime}=H_{p_{r}}, \quad p^{\prime}=-k p, \quad p_{r}^{\prime}=-H_{q_{r}}-k p_{r}, \quad z^{\prime}=p+p_{r} H_{p_{r}},
$$

They possess the integral

$$
\left({ }^{\prime}=d / d u\right) \text {. }
$$

$$
p+k z+H\left(q_{r}, p_{s}\right)=\text { const. }
$$

By adjoining

$$
t=u, \quad p=p_{0} e^{-k\left(t-t_{0}\right)}, \quad z=z_{0}+\int_{t_{0}}^{t}\left(p_{r} H_{p_{r}}+p\right) d t,
$$

to the equations of a motion of (3), one obtains a solution of (8). Taking $p_{0}$ arbitrarily, $z_{0}$ is determined so that the constant in (9) equals zero, and the relation obtained is used to eliminate $p$ from the last equation in (8) to obtain

$$
d z / d t+k z=p_{r} H_{p_{r}}-H,
$$

which, when integrated, yields (7). The theorem then follows from (9).

CoROllary. If $H$ is homogeneous of degree two in $q_{r}, p_{s}$, a quasi-conservative system has a first integral $\left(H+(k / 2) p_{r} q_{r}\right) e^{k t}=$ const.

To establish the corollary, set $2 H=q_{r} H_{q_{r}}+p_{r} H_{p_{r}}$ in (7) and substitute for $H_{q_{r}}, H_{p_{r}}$ from (3) to obtain $2 e^{k t} S=e^{k t} p_{r} q_{r}+$ const.

The generalization of the principle of least action to quasi-conservative systems rests on the following lemma $\left({ }^{10}\right)$.

LEммA. The projections of the characteristic curves of the partial differential equation

$$
f\left(x_{r}, z, p_{s}\right)=0, \quad p_{s}=z_{x_{s}}, f_{p_{1}}^{2}+\cdots+f_{p_{n}}^{2} \neq 0, \operatorname{det}\left\|f_{p_{r} p_{s}}\right\| \neq 0,
$$

upon the space of the independent variables $x_{r}$ are the extremals of the Mayer calculus of variations problem $\delta z_{1}=0$, given that

$$
d z / d t=F\left(x_{r}, z, \dot{x}_{s}\right), \quad \delta x_{r}^{0}=\delta z^{0}=0, \quad \delta x_{r}^{1}=0,
$$

where $\zeta-z=F\left(x_{r}, z, x_{s}-\xi_{s}\right)$ is the equation of the Monge cone for the partial differential equation at the point $x_{r}, z$.

THE PRINCIPLE OF LEAST ACTION. The projections of the characteristic curves of the partial differential equation $k z+H\left(q_{r}, p_{s}\right)=0, p_{s}=z_{q_{s}}$ upon the space of the coordinates $q_{r}$ are the extremals of the Mayer calculus of variations problem $\delta z_{1}=0$, given that

$$
\dot{z}=\left(2(c-k z) a_{r s} \dot{q}_{r} \dot{q}_{s}\right)^{1 / 2}+b_{r} \dot{q}_{r}, \quad \delta q_{r}^{0}=\delta z^{0}=0, \quad \delta q_{r}^{1}=0 .
$$

(10) For the proof of this lemma for the partial differential equation $f(x, y, p, q)=0$, see A. Kneser, Lehrbuch der Variationsrechnung, Braunschweig, 1925, pp. 157-160. 
To arrive at the principle, we set $S=h e^{-k t}+W$, where $h=$ const. and $W=W\left(q_{1}, \cdots, q_{n}\right)$ in (4) to obtain the partial differential equation $k W+H\left(q_{r}, W_{q_{s}}\right)=0$. Placing $z=W, p_{s}=W_{q_{s}}$ and calculating the equation of the Monge cone for this partial differential equation, the truth of the principle follows from the lemma.

To see why the principle is to be regarded as a generalization of the principle of least action for conservative systems, it will be recalled that placing $S=-h t+W$ in the Hamilton-Jacobi partial differential equation of a conservative system leads to the partial differential equation $-h+H\left(q_{r}, W_{q_{s}}\right)=0$. The projections upon the space of the coordinates $q_{r}$ of the characteristics of this partial differential equation are the extremals of the Mayer calculus of variations problem $\delta z^{1}=0$, given that

$$
\dot{z}=\left(2(c+h) a_{r s} \dot{q}_{r} \dot{q}_{s}\right)^{1 / 2}+b_{r} \dot{q}_{r}, \quad \delta q_{r}^{0}=\delta z^{0}=0, \quad \delta q_{r}^{1}=0 .
$$

When this is formulated as an ordinary calculus of variations problem with fixed end points

$$
\delta \int_{t_{0}}^{t_{1}}\left\{\left(2(\varepsilon+h) a_{r s} \dot{q}_{r} \dot{q}_{s}\right)^{1 / 2}+b_{r} \dot{q}_{r}\right\} d t=0,
$$

one obtains the classic expression for the principle of least action.

2. Characteristic exponents $\left({ }^{11}\right)$. Assuming that the origin of the phase space is an equilibrium motion of (3), the equations of variation written in matrix form are

$$
d \mathrm{x} / d t=A \mathrm{x}, \quad \boldsymbol{A}=\mathrm{GH}-k \mathrm{~K} .
$$

Here $\mathrm{x}$ denotes a matrix with $2 n$ rows and one column, the first and last $n$ rows being occupied by the variations of $q_{r}, p_{s}$, respectively, while ( ${ }^{12}$ )

$\boldsymbol{G}=\left\|\begin{array}{rr}\omega & \varepsilon \\ -\varepsilon & \omega\end{array}\right\|, \quad H=\left\|\begin{array}{ll}H_{q_{r} q_{s}} & H_{q_{r} p_{s}} \\ H_{r_{r} q_{s}} & H_{p_{r} p_{s}}\end{array}\right\|, \quad K=\left\|\begin{array}{cc}\omega & \omega \\ \omega & \varepsilon\end{array}\right\|, \quad M=\left\|\begin{array}{cc}\omega & \varepsilon \\ \varepsilon & \omega\end{array}\right\|$,

where $\omega, \varepsilon$ denote the $n \times n$ zero and unit matrices, respectively, and the partial derivatives are evaluated at the origin. Denoting the transposed matrix by affixing a prime, one verifies that

$$
\begin{aligned}
& G^{\prime}=-G, \quad G G^{\prime}=G^{\prime} G=-G^{2}=E ; \\
& H^{\prime}=H, \quad K^{\prime}=K, \quad G K G=K-E,
\end{aligned}
$$

where $E$ is the unit matrix of $2 n$ rows and columns.

(11) For the theorems on characteristic exponents of conservative systems, see G. D. Birkhoff, Dynamical Systems, loc. cit., pp. 74-78, and A. Wintner, Three notes on characteristic exponents and equations of variation in celestial mechanics, American Journal of Mathematics, vol. 53 (1931), p. 609.

(12) The matrix $M$ defined here will not be needed until Theorem 4 . 
THEOREM 3. The characteristic exponents may be divided into pairs in which the two members are of equal multiplicity and have $-k$ for their sum.

The characteristic exponents are the roots of the equation $f(\lambda)$ $=\operatorname{det}(A-\lambda E)=0$. Replacing $A$ by its transposed, multiplying before and behind by $G$, it follows from (11) that $f(\lambda)=f(-\lambda-k)$, and the theorem is proved.

It may be noted in passing that at least half of the characteristic exponents have negative (positive) real parts if $k>0(k<0)$.

Theorem 4. If the matrix $H+(k / 2) M$ is definite, the characteristic exponents lie on the line $\Re(\lambda)=-k / 2$ with $\lambda=-k / 2$ excluded.

Corresponding to a characteristic exponent $\lambda$, there is a solution of (10) given by $\mathbf{x}=\boldsymbol{a} e^{\lambda t}$, where $\boldsymbol{a}$ is a constant matrix satisfying $(A-\lambda E) \boldsymbol{a}=0$. Multiplying on the left by $a^{\prime} G$ and observing that $2 G K=M+G$, it is found that

$$
\boldsymbol{a}^{\prime}(\boldsymbol{H}+(k / 2) \boldsymbol{M}) \boldsymbol{a}=\mathbf{0},
$$

inasmuch as $G$ is skew-symmetric. If $\lambda$ is real, $a$ may be taken real and the assumption that $H+(k / 2) M$ is definite is contradicted by (12). Thus no characteristic exponent is real.

We may regard (10) as the canonical equations of a quasi-conservative system whose Hamiltonian function $H$ is homogeneous of degree 2 in its arguments and write the first integral, previously obtained in the corollary to Theorem 1, in the matrix form $\mathbf{x}^{\prime}(\boldsymbol{H}+(k / 2) \boldsymbol{M}) \mathbf{x} \boldsymbol{e}^{k t}=$ const. For complex $\lambda$, the real solution $x+\bar{x}$ is formed and inserted in the energy integral. Keeping (12) in mind, one finds that $\boldsymbol{a}^{\prime}(\boldsymbol{H}+(k / 2) \boldsymbol{M}) \overline{\boldsymbol{a}} \exp (k+\lambda+\bar{\lambda}) t=$ const., which requires $\Re(\lambda)=-k / 2$.

\section{NATURAL QUASI-CONSERVATIVE SYSTEMS}

A quasi-Lagrangian system (3) is a natural system if $b_{r}=0$. Taking $b_{r}=0$ in (5), it follows from Theorem 1 that the $\omega$-limiting motions of a positively stable motion of a natural quasi-conservative system (3) are equilibrium motions of (3). Such systems accordingly possess no periodic motions.

THEOREM 5. To each characteristic constant $\delta_{r}$ of the matrix $\left\|-c_{q_{r} q_{s}}\right\|$, evaluated at an equilibrium motion of (3), there correspond two characteristic exponents given by the roots of the equation

$$
\lambda^{2}+k \lambda+\delta_{r}=0 .
$$

Referring to $\S 2$ of II, it is apparent that

$$
\boldsymbol{H}=\left\|\begin{array}{cc}
\boldsymbol{\beta} & \omega \\
\omega & \alpha
\end{array}\right\|, \quad \boldsymbol{\alpha}=\left\|a^{r \xi}\right\|, \quad \boldsymbol{\beta}=\left\|-c_{q_{r} q_{\mathrm{s}}}\right\|,
$$

and it may be verified that the transformation $x=T y$, where 


$$
T=\left\|\begin{array}{ll}
\gamma & \omega \\
\omega & \gamma^{\prime-1}
\end{array}\right\|, \quad T^{\prime} H T=\left\|\begin{array}{cc}
\gamma^{\prime} \widehat{\zeta} & \omega \\
\omega & \gamma^{-1} \alpha \gamma^{\prime-1}
\end{array}\right\|,
$$

carries (10) into $d y / d t=B y$, with $B=G T^{\prime} H T-k K$. Choosing $\gamma$ so that $\gamma^{\prime} \alpha^{-1} \gamma=\varepsilon, \gamma^{\prime} \underline{\gamma}=\delta$ where $\delta$ is a diagonal matrix with the characteristic constants of $\boldsymbol{\beta}$ along the principal diagonal, the theorem follows inasmuch as the characteristic exponents satisfy the equation $\operatorname{det}(B-\lambda E)=0$.

It is clear that there are no purely imaginary characteristic exponents. If the characteristic exponents $\lambda_{1}, \cdots, \lambda_{m}$ have negative real parts and the remaining $2 n-m$ have positive real parts, the equilibrium motion is of negative or positive general type, according as none of the linear commensurability relations

$$
\begin{array}{r}
\text { I }\left\{\begin{array}{lr}
p_{1} \lambda_{1}+\cdots+p_{m} \lambda_{m}=\lambda_{j}, & j=1, \cdots, m ; \\
p_{1}+\cdots+p_{m} \geqq 2, & p_{j}=0,1, \cdots ;
\end{array}\right. \\
\mathrm{III}\left\{\begin{array}{lr}
p_{m+1} \lambda_{m+1}+\cdots+p_{2 m} \lambda_{2 m}=\lambda_{k}, \\
p_{m+1}+\cdots+p_{2 m} \geqq 2, & k=1, \cdots, 2 n ;
\end{array}\right.
\end{array}
$$

in I or II is satisfied.

THEOREM 6. If an equilibrium motion $M_{0}$ is of negative [positive] general type, a suitably restricted neighborhood of $M_{0}$ contains an analytic m-dimensional $[(2 n-m)$-dimensional $]$ surface, the points of which tend towards $M_{0}$ as a limit as $t \rightarrow+\infty[-\infty]$. No other points of this neighborhood tend towards $M_{0}$ as a limit as $t \rightarrow+\infty[-\infty]$. The number $m$ is the number of characteristic exponents of $M_{0}$ with negative real parts $\left.{ }^{(13}\right)$.

\section{THE RESTRICTED PROBLEM OF THREE BODIES}

Two finite masses $\mu$ and $1-\mu$ move in a fixed plane under their mutual gravitational attraction. The restricted problem of three bodies is the problem of determining the motion in the fixed plane of a third mass (the infinitesimal mass) subject to the gravitational attraction of the two finite masses, the motion of which is assumed to proceed independently of its presence. The problem is said to be of elliptic, parabolic or hyperbolic type according as the orbit of $\mu$ about $1-\mu$ is an ellipse, parabola or hyperbola. When the conic section degenerates into a line segment the problem is termed rectilinear, otherwise we term the problem general.

If we take the center of mass of the two finite masses as origin of a rectangular coordinate system $(\xi, \eta)$ having a fixed orientation in the plane of motion of the two finite masses, it is known that

(14) $\rho^{\prime \prime}-\rho \theta^{\prime 2}=-\kappa^{2} \rho^{-2}, \rho^{2} \theta^{\prime}=\alpha=$ const., $\quad\left(\kappa^{2}=\right.$ gravitational constant $)$,

(13) A proof of this theorem has been given by the writer in Bulletin of the American Mathematical Society, vol. 46 (1940), pp. 475-481. See also C. L. Siegel, Der Dreierstoss, Annals of Mathematics, (2), vol. 42 (1941), pp. 156-165. 
where $\rho, \theta$, respectively, denote the length and angle of inclination with the positive $\xi$-axis of the line joining the two finite masses, differentiation with respect to the time $\tau$ being indicated by a prime. Denoting the distances of the infinitesimal mass from $1-\mu, \mu$ by $\rho_{1}, \rho_{2}$, respectively, the Lagrangian function $\Lambda$ for its motion is

$$
\Lambda=\left(\xi^{\prime 2}+\eta^{\prime 2}\right) / 2+\kappa^{2}\left((1-\mu) / \rho_{1}+\mu / \rho_{2}\right) .
$$

THEOREM 7. The introduction of new variables $x, y, t$ by

$$
\xi+i \eta=\rho e^{i \theta}(x+i y), \quad \kappa d \tau=\rho^{3 / 2} d t, \quad \rho^{3 / 2}>0,
$$

reduces the restricted problem of three bodies to a quasi-Lagrangian system in which

$$
\begin{array}{ll}
L=\left(\dot{x}^{2}+\dot{y}^{2}\right) / 2+b e^{-l}(x \dot{y}-y \dot{x})+\Omega, & b=\alpha / \kappa, \\
H=\left[\left(p+b e^{-l} y\right)^{2}+\left(q-b e^{-l} x\right)^{2}\right] / 2-\Omega, & l=(\log \rho) / 2,
\end{array}
$$

where

$$
\begin{gathered}
\Omega=\left(x^{2}+y^{2}\right) / 2+(1-\mu) / r_{1}+\mu / r_{2}, \\
r_{1}^{2}=(x+\mu)^{2}+y^{2}, \quad r_{2}^{2}=(x-1+\mu)^{2}+y^{2},
\end{gathered}
$$

and differentiation with respect to $t$ is indicated by a dot. The system is acquisitive or dissipative according as the two finite masses approach or leave each other and reduces to a natural system for rectilinear problems.

It may be verified that

$$
\begin{aligned}
\xi^{\prime 2}+\eta^{\prime 2}= & \rho^{2}\left(x^{\prime 2}+y^{\prime 2}\right)+2 \rho^{2} \theta^{\prime}\left(x y^{\prime}-y x^{\prime}\right) \\
& +\left(\rho^{\prime 2}+\rho^{2} \theta^{\prime 2}\right)\left(x^{2}+y^{2}\right)+2 \rho \rho^{\prime}\left(x x^{\prime}+y y^{\prime}\right), \\
{\left[\rho \rho^{\prime}\left(x^{2}+y^{2}\right)\right]^{\prime}=} & \left(\rho^{\prime 2}+\rho \rho^{\prime \prime}\right)\left(x^{2}+y^{2}\right)+2 \rho \rho^{\prime}\left(x x^{\prime}+y y^{\prime}\right) .
\end{aligned}
$$

When these equations are subtracted with (14) in mind, the result substituted in $\Lambda$, and the term $\left[\rho \rho^{\prime}\left(x^{2}+y^{2}\right)\right]^{\prime}$ suppressed( $\left.{ }^{14}\right)$, it is found that

$$
\Lambda=\left(\rho^{2} / 2\right)\left(x^{\prime 2}+y^{\prime 2}\right)+\alpha\left(x y^{\prime}-y x^{\prime}\right)+\left(\kappa^{2} / \rho\right) \Omega,
$$

provided one observes that $\rho_{1}=\rho r_{1}, \rho_{2}=\rho r_{2}$.

Writing the equations of motion for the infinitesimal mass in the variational form $\delta \int_{\tau_{0}}^{\tau_{1}} \Lambda d \tau=0$ and introducing the new independent variable $t$ defined in (15), this variational equation takes the form (2) with $l, L$ defined as in (16).

It will be observed that the Hamiltonian function $H$ has the first form in (5). The system is therefore acquisitive or dissipative as stated.

(14) This may be done since the variation of its integral vanishes. 
REMARK. If the new independent variable is defined by $\kappa d \tau=\rho d \bar{t}$ the restricted problem of three bodies reduces to a quasi-Lagrangian system with

$$
\begin{aligned}
L & =\left(\dot{x}^{2}+\dot{y}^{2}\right) / 2+b e^{-l}(x \dot{y}-y \dot{x})+e^{-l} \Omega, & b=\alpha / \kappa, \\
H & =\left[\left(p+b e^{-l} y\right)^{2}+\left(q-b e^{-l} x\right)^{2}\right] / 2-e^{-l} \Omega, & l=\log \rho,
\end{aligned}
$$

where the dot denotes differentiation with respect to $\bar{t}$. The Hamiltonian function has the second form in (5), the system being acquisitive or dissipative according as the finite masses approach or leave each other, and is a natural system for rectilinear problems.

\section{THE RESTRICTED PROBLEM OF PARABOLIC TYPE}

1. The differential equations of motion. If $\mu$ moves in a parabolic orbit about $1-\mu$, it is $\operatorname{known}\left({ }^{15}\right)$ that

$$
\rho=(p / 2) \sec ^{2}(\theta / 2), \quad 2 \kappa \tau=p^{3 / 2}\left((1 / 3) \tan ^{3}(\theta / 2)+\tan (\theta / 2)\right), p>0,
$$

where $p$ is the semi-latus rectum of the parabolic orbit. In case $p=0$, the parabolic orbit degenerates into a line segment, and we have

$$
\rho=\left(3 \kappa \tau / 2^{1 / 2}\right)^{2 / 3} .
$$

When the variable $t$ of Theorem 7 is introduced in place of $\tau$, these equations are replaced by

$$
\begin{aligned}
& \rho=(p / 2) \cosh ^{2}\left(t / 2^{1 / 2}\right), \quad \sin (\theta / 2)=\tanh \left(t / 2^{1 / 2}\right), \\
& \rho=e^{ \pm 2^{1 / 2} t} .
\end{aligned}
$$

Thus, as $t$ ranges from $-\infty$ to $+\infty$, the mass $\mu$ describes the complete parabolic orbit $(p>0)$ or describes the line segment $(p=0)$, approaching a collision with $1-\mu$ as $t \rightarrow \pm \infty$ according as the negative or positive sign is taken in (22). Corresponding to (21), (22) the function $l$ in (16) is given by

$$
l=(1 / 2) \log (p / 2)+\log \cosh \left(t / 2^{1 / 2}\right), \quad l= \pm t / 2^{1 / 2},
$$

from which the differential equations (1) are found, on setting $c=b(2 / p)^{1 / 2}$

$$
\begin{aligned}
& \ddot{x}+\left(1 / 2^{1 / 2}\right) \tanh \left(t / 2^{1 / 2}\right) \cdot \dot{x}-2 c \operatorname{sech}\left(t / 2^{1 / 2}\right) \cdot \dot{y}=\Omega_{x}, \\
& \ddot{y}+\left(1 / 2^{1 / 2}\right) \tanh \left(t / 2^{1 / 2}\right) \cdot \dot{y}+2 c \operatorname{sech}\left(t / 2^{1 / 2}\right) \cdot \dot{x}=\Omega_{y}, \\
& \ddot{x} \pm \dot{x} / 2^{1 / 2}=\Omega_{x}, \quad \ddot{y} \pm \dot{y} / 2^{1 / 2}=\Omega_{y},
\end{aligned}
$$

the latter equations holding for the rectilinear problem, with the positive or negative sign taken according as the finite masses leave or approach each other. The canonical form (3) of (23) is

$$
\dot{x}=H_{p}, \quad \dot{y}=H_{x}, \quad \dot{p}=-H_{x}-k p, \quad \dot{q}=-H_{y}-k q,
$$

(15) See, for example, F. R. Moulton, An Introduction to Celestial Mechanics, New York, 1935, pp. 155-159. 
where $k=\left(1 / 2^{1 / 2}\right) \tanh \left(t / 2^{1 / 2}\right)$ and $H$ is defined in (16), while that of (24) is

$$
\begin{aligned}
\dot{x}=\bar{H}_{p}, \quad \dot{y} & =\bar{H}_{q}, \quad \dot{p}=-\bar{H}_{x}-\bar{k} p, \quad \dot{q}=-\bar{H}_{y}-\bar{k} q, \\
\bar{H} & =\left(p^{2}+q^{2}\right) / 2-\Omega, \quad \bar{k}= \pm 1 / 2^{1 / 2} .
\end{aligned}
$$

Corresponding to the positive and negative values for $\bar{k}$ we have two kinds of rectilinear problems; the former is termed the dissipative rectilinear problem and the latter the acquisitive rectilinear problem.

2. The flow in the phase space. A comparison of (25) and (26) shows that the $\alpha$ and $\omega$-limiting motions in the general problem are, respectively, motions of the acquisitive and dissipative rectilinear problems.

The set $S$ of singularities of the system (25) comprises the points of the two-dimensional surfaces $x=-\mu, y=0 ; x=1-\mu, y=0$ corresponding to collisions of the infinitesimal mass with one or the other of the finite masses.

THEOREM 8. If the energy $H$ of a motion tends to $-\infty$ as $t \rightarrow+\infty$ the motion is positively instable and the point $P$ in the $(x, y)$-plane corresponding to the infinitesimal mass either tends uniformly towards one of the points corresponding to the finite masses or else tends uniformly towards the point at infinity as $t \rightarrow+\infty$.

Suppose that $P$ tends uniformly to neither of the points corresponding to the finite masses nor to the point at infinity as $t \rightarrow+\infty$. There would exist a positive $\epsilon$ and an infinite sequence $\left\{t_{n}\right\}$ of $t$-values for which $P$ would lie in the region

$$
(x+\mu)^{2}+y^{2}>\epsilon, \quad(x-1+\mu)^{2}+y^{2}>\epsilon, \quad x^{2}+y^{2}>1 / \epsilon,
$$

and from the definitions of $H, \bar{H}$ in (16), (26) there would exist a constant $K$ such that $H\left(t_{n}\right)>K$, thus contradicting the hypothesis.

The equilibrium motions of the rectilinear problems are characterized by

$$
p=0, \quad q=0, \quad \Omega_{x}=0, \quad \Omega_{y}=0,
$$

the latter two equations being satisfied at exactly five points $L_{i}$ in the $(x, y)$ plane known as libration points. $L_{1}, L_{2}, L_{3}$ lie on the $x$-axis with $L_{1}$ between, $L_{2}$ to the right of, and $L_{3}$ to the left of the points corresponding to the finite masses which in turn form equilateral triangles with $L_{4}, L_{5}$. Corresponding to $L_{i}$ there are five equilibrium motions $E_{i}$ in the phase space.

THEOREM 9. If the point $P$ representing the infinitesimal mass remains in a bounded closed region of the $(x, y)$-plane containing $L_{i}$ and not containing the points corresponding to the finite masses, it tends uniformly towards a definite $L_{i}$ as $t \rightarrow \pm \infty$.

In view of the restriction upon $P$ it is clear that $-\Omega$ has a finite lower bound. Since $H$ decreases monotonely for sufficiently large $t$, it follows that 
$p^{2}+q^{2}$ remains bounded for $t>t_{0}$. The corresponding motion $M$ in the phase space is accordingly stable and it follows from Theorem 1 that the $\omega$-limiting motions of $M$ are the equilibrium motions $E_{i}$ of (26). Now $M$ approaches its set of $\omega$-limiting motions uniformly and therefore it approaches a definite $E_{i}$ uniformly as $t \rightarrow+\infty$.

The proof of the theorem has been given only for the general problem. It is evident that it remains in force for the rectilinear problem and when $t \rightarrow-\infty$.

Since a stable motion has one $E_{i}$ as a unique $\alpha$-limiting point and one as a unique $\omega$-limiting point, the stable motions may be divided into twenty-five classes, inasmuch as the $\alpha$ - and $\omega$-limiting points conceivably may be chosen at random from the five $E_{i}$.

3. The rectilinear problem. The differential equations (24) for the acquisitive and dissipative rectilinear problems interchange when $t$ is replaced by $-t$. It will be sufficient, therefore, to consider one of these problems. We shall select the dissipative problem for further investigation.

TheOREM 10. If stable motions other than equilibrium motions exist in the dissipative rectilinear problem, they fall into the following nine classes:

(i) $\alpha$-limiting point $E_{4}$ or $E_{5}$, $\omega$-limiting point $E_{1}, E_{2}, E_{3}$;

(ii) $\alpha$-limiting point $E_{3}$,

(iii) $\alpha$-limiting point $E_{2}$, $\omega$-limiting point $E_{1}, E_{2}$; $\omega$-limiting point $E_{1}$;

provided $0<\mu<1 / 2$. When $\mu=1 / 2$ there is no motion in (ii) with $E_{2}$ as $\omega$-limit point and when $1 / 2<\mu<1$, the roles of $E_{2}, E_{3}$ are interchanged.

Along a motion $M$ of (26) not an equilibrium motion $\bar{H}$ decreases monotonely with increasing $t$ for all $t$. Therefore an $E_{i}$ cannot serve simultaneously as $\alpha$ - and $\omega$-limiting point for $M$, since this would require $\bar{H}$ constant along $M$ and $d \bar{H} / d t=-\left(p^{2}+q^{2}\right) / 2^{1 / 2} \equiv 0$ would imply that $M$ is an equilibrium motion, contrary to hypothesis.

If $\Omega_{i}$ is the value of $\Omega$ at $L_{i}$, it is known $\left({ }^{16}\right)$ that $\Omega_{2}$ is greater than, equal to, or less than $\Omega_{3}$ according as $\mu$ is less than, equal to, or greater than $1 / 2$ and that $\Omega_{1}$ exceeds while $\Omega_{4}=\Omega_{5}$ is less than either of $\Omega_{2}, \Omega_{3}$. Therefore, if $\bar{H}_{i}$ indicates the value of $\bar{H}$ at $E_{i}$ it follows that $\bar{H}_{2}$ is less than, equal to, or greater than $\bar{H}_{3}$ according as $\mu$ is less than, equal to, or greater than $1 / 2$ and that $\bar{H}_{1}$ is less than while $\bar{H}_{4}=\bar{H}_{5}$ is greater than either $\bar{H}_{2}$ or $\bar{H}_{3}$.

It is therefore impossible for a stable motion to have one $E_{4}, E_{5}$ as $\alpha$-limiting point and the other for $\omega$-limiting point and the theorem is an immediate consequence of the above inequalities and the monotone character of $\bar{H}$.

A study of the nature of the flow in the phase space in the neighborhoods of $E_{i}$ leads to a sharper classification of stable motions. Prior to such study a lemma dealing with the characteristic exponents of the $E_{i}$ is needed.

(18) See A. Wintner, The Analytical Foundations of Celestial Mechanics, Princeton, 1941, pp. 364-366. 
LEMMA. There is one positive and one negative characteristic exponent for $E_{1}$; the two remaining are conjugate complex numbers with negative real parts. There is one positive characteristic exponent for $E_{2}$; the remaining three have negative real parts and there exists a constant $\mu^{*}$ such that of these three: one is real and the other two are conjugate complex numbers for $0<\mu<\mu^{*}$; all are real with two equal to $-2^{-3 / 2}$ and differing from the third if $\mu=\mu^{*}$, all are real and different for $\mu^{*}<\mu<1$. At $E_{3}$ the situation is analogous to that at $E_{2}$ and at $E_{4}, E_{5}$ there are two distinct positive and two distinct negative characteristic exponents.

Consider the three libration points $L_{i}\left(a_{i}, 0\right)$ on the $x$-axis. One finds that

$$
\Omega_{x x}\left(a_{i}, 0\right)=1+2 A_{i}, \quad \Omega_{x y}\left(a_{i}, 0\right)=0, \quad \Omega_{y y}=1-A_{i},
$$

where

$$
A_{i}=\frac{1-\mu}{\left|a_{i}+\mu\right|^{3}}+\frac{\mu}{\left|a_{i}+\mu-1\right|^{3}} .
$$

If we use (27) to compute the characteristic constants of the matrix $\left\|-c_{q_{r} q_{s}}\right\|$ in Theorem 5 , and take $k=1 / 2^{1 / 2}$ in this theorem, the characteristic exponents $\lambda$ of $E_{i}$ turn out to be

$$
\lambda=2^{-3 / 2}\left(-1 \pm\left(9+16 A_{i}\right)^{1 / 2}\right), \quad \lambda=2^{-3 / 2}\left(-1 \pm\left(9-8 A_{i}\right)^{1 / 2}\right),
$$

from which the statements of the theorem relative to the characteristic exponents at $E_{1}, E_{2}, E_{3}$ follow, inasmuch as it may be proved that $A_{1}>4$ and $A_{2}\left(A_{3}\right)$ decreases (increases) monotonely and continuously from 4 (1) to 1 (4) as $\mu$ varies from 0 to 1 .

After computing the partial derivatives of second order for $\Omega$ at $L_{4}, L_{5}$ and obtaining the characteristic constants of $\left\|-c_{q_{r} q_{s}}\right\|$, it is found that the characteristic exponents $\lambda$ of $E_{4}, E_{5}$ are given by

$$
\lambda=2^{-3 / 2}\left(-1 \pm\left(13 \pm 12(1-3 \mu(1-\mu))^{1 / 2}\right)^{1 / 2}\right),
$$

from which follow the properties for the characteristic exponents of $E_{4}, E_{5}$.

In view of this lemma, Theorem $6(n=2, m=3)$ applies to $E_{1}$ for all values of $\mu$, to $E_{2}$ if $\mu \neq \mu^{*}$, to $E_{3}$ provided $\mu \neq 1-\mu^{*}$ and $(m=2)$ to $E_{4}, E_{5}$ for all values of $\mu . E_{1}, E_{2}, E_{3}$ are of positive general type, since none of the linear commensurability relations II of (13) is fulfilled.

THEOREM 11. In a suitably restricted neighborhood of an $E_{i}(i=1,2,3)$ of negative general type the locus of points which lie on positively [negatively] stable motions having $E_{i}$ as a unique $\omega-[\alpha-]$ limiting point is an analytic hypersurface [curve]. In a suitably restricted neighborhood of an $E_{i}(i=4,5)$ of negative [positive] general type the locus of points which lie on positively [negatively] stable motions having $E_{i}$ for a unique $\omega$ - $[\alpha-]$ limiting point is an analytic two-dimensional manifold. to six.

The number of classes of stable motions may now be reduced from nine 
THEOREM 12. Excluding the values $\mu^{*}, 1-\mu^{*}$ of $\mu$, if stable motions other than equilibrium motions exist, the $\alpha$-limiting point is $E_{4}$ or $E_{5}$ and the w-limiting point is one of $E_{1}, E_{2}, E_{3}$.

To prove the theorem we show that there are no stable motions in the classes (ii), (iii) of Theorem 10.

The motions in the phase space for which the infinitesimal mass remains on the line joining the finite masses lie in the $(x, p)$-plane and are solutions of the differential system

$$
\dot{x}=p, \quad \dot{p}=\Omega_{x}-p / 2^{1 / 2} .
$$

The flow in the $(x, p)$-plane has three equilibrium motions $\left(a_{i}, 0\right)$ $(i=1,2,3)$ separated by the points $(-\mu, 0),(1-\mu, 0)$ corresponding to the finite masses. One characteristic constant is positive and the other negative at each equilibrium motion. It follows from Theorem 6 that the locus of points in a sufficiently small neighborhood of $\left(a_{i}, 0\right)$ lying upon motions of (28) having $\left(a_{i}, 0\right)$ as a unique $\alpha$-limiting point is an analytic curve $\left({ }^{17}\right)$ through $\left(a_{i}, 0\right)$. These equilibrium motions appear in the phase space of (26) as $E_{i}$ $(i=1,2,3)$, and since the motions of (26) having $E_{i}$ as a unique $\alpha$-limiting point are confined to an analytic curve $\left({ }^{17}\right)$ through $E_{i}$, they must lie entirely in the $(x, p)$-plane.

A stable motion of (26) with one $E_{1}, E_{2}, E_{3}$ as $\alpha$-limiting point and another as $\omega$-limiting point is therefore impossible, for such a stable motion would require the infinitesimal mass to collide with one of the finite masses.

4. The existence of stable motions. For Theorem 12 to have content a demonstration of the existence of stable motions other than equilibrium motions is essential. We shall show that two stable motions exist when $\mu=1 / 2$. Whether other stable motions exist for $\mu=1 / 2$, or whether any stable motions exist when $\mu \neq 1 / 2$, are unsolved problems.

Setting $\mu=1 / 2, x=p=0$ in (26) we obtain a differential system of the second order

$$
\dot{y}=q, \quad \dot{q}=\phi(y)-q / 2^{1 / 2}, \quad \phi(y)=y\left[1-\left((1 / 4)+y^{2}\right)^{-3 / 2}\right],
$$

for the motions in the phase space when the infinitesimal mass is restricted to lie on the perpendicular bisector of the line segment connecting the two finite masses.

Since $\phi$ is an odd function of $y$, the motions in the $(y, q)$-plane are paired, the members of a pair being symmetric to each other with respect to the origin. The graph $G$ of $q=2^{1 / 2} \phi$ is indicated by $A B C O C^{\prime} B^{\prime} A^{\prime}$ in Figure 1. It rises monotonely along $A B C, C^{\prime} B^{\prime} A^{\prime}$ and falls monotonely along $C O C^{\prime}$. The flow proceeds to the right [left] in the upper [lower] half-plane, being vertical on the $y$-axis, and is directed downwards [upwards] in the region above [un-

(17) The curve has no multiple point at the equilibrium motion. 
derneath] $G$, being horizontal on $G$, except for the equilibrium motions $B, O, B^{\prime}$ corresponding to $E_{5}, E_{1}, E_{4}$.

The characteristic exponents of $O$ are congugate complex numbers with negative real parts. It follows that, sufficiently near to $O$, the motions have a spiral character ${ }^{18}$ ) about $O$, that is, as $t \rightarrow+\infty$ the point $P$ on the motions tends towards $O$ with the angle between $O P$ and the $y$-axis increasing indefinitely.

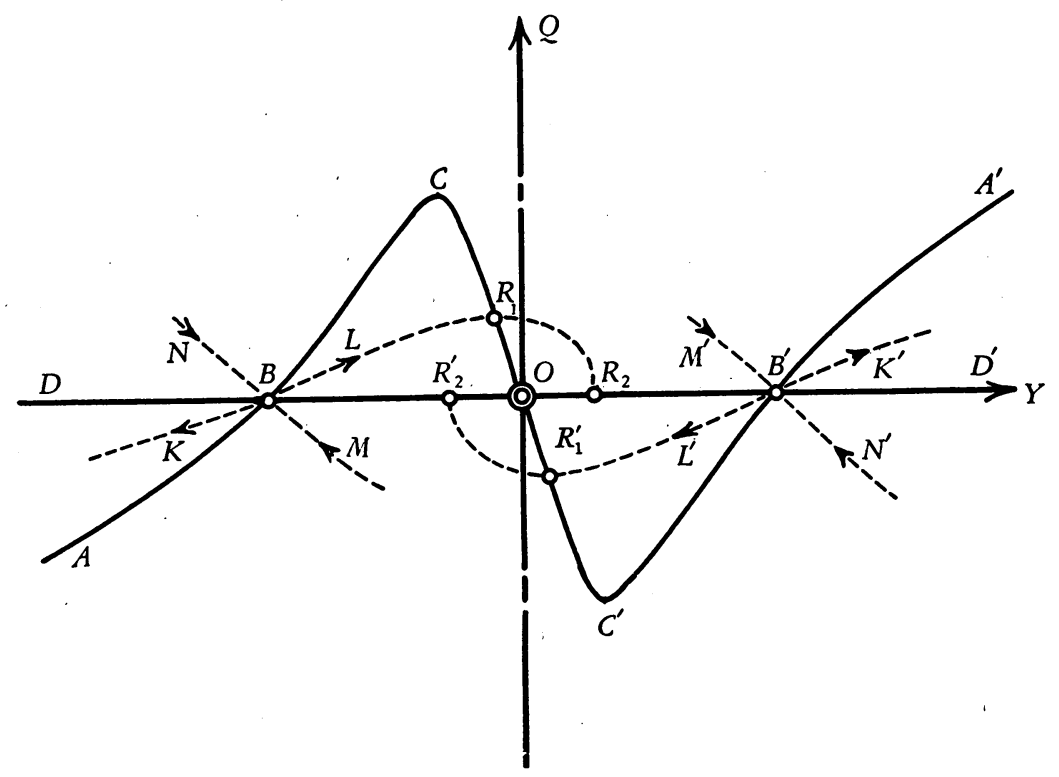

FIG. 1.

At $B^{\prime}$ one characteristic exponent is positive and the other is negative. Through $B^{\prime}$ there accordingly pass two analytic arcs $K^{\prime} B L^{\prime}, M^{\prime} B N_{.}^{\prime}$, the loci of points lying on motions having $B^{\prime}$ for unique $\alpha$-, $\omega$-limiting point, respectively. Upon calculation it is found that these arcs are disposed with respect to $G$ as shown in Figure 1 .

Consider the arc $B^{\prime} L^{\prime}$ which in the immediate neighborhood of $B^{\prime}$ lies in the open region $B^{\prime} O C^{\prime} B^{\prime}$. In this region the flow is directed to the left and downwards, and since the region contains no equilibrium motion upon which the arc could end, it leaves the region by way of a point $R_{1}^{\prime}$ on the arc $O C^{\prime}$ to enter the open region in the lower half-plane under $G$. Here the flow is upwards and to the left and, since the region contains no equilibrium points, the arc $B^{\prime} L^{\prime} R_{1}^{\prime}$ must leave it by: (a) the open arc $R_{1}^{\prime} O$, (b) $O$, (c) the open segment $O B$ of the $y$-axis, (d) $B$, (e) the open arc $B A$. Clearly (a) is impossible, since on $R_{1}^{\prime} O$ the flow is horizontal and directed from right to left. We may

(18) See, for example, L. Bierberbach, Differentialgleichungen, Berlin, 1930, pp. 104-105. 
exclude (b) in view of the spiral character of the flow about $O$. The possibility (c) is illustrated in Figure 1 by $B^{\prime} L^{\prime} R_{1}^{\prime} R_{2}^{\prime}$. Paired with such a motion, there is a motion on the arc $B L R_{1} R_{2}$ symmetric to it with respect to $O$. The two arcs $B L R_{1} R_{2}, B^{\prime} L^{\prime} R_{1}^{\prime} R_{2}^{\prime}$ and the segments $B R_{2}^{\prime}, B^{\prime} R_{2}$ of the $y$-axis enclose a region containing $O$ into which the motion on $B^{\prime} L^{\prime} R_{1}^{\prime} R_{2}^{\prime}$ enters and never leaves. It cannot leave by way of the open segments $B R_{2}^{\prime}, B^{\prime} R_{2}$, for on the former the flow is vertically upwards and on the latter vertically downwards. Departure by way of $B$ or $B^{\prime}$ is ruled out by Theorem 10 and it cannot meet either $B L R_{1} R_{2}$ or $B^{\prime} L^{\prime} R_{1}^{\prime} R_{2}^{\prime}$ for such a point of meeting can occur only at an equilibrium motion. The motion accordingly remains in this region and is therefore stable. Paired with it, there exists a second stable motion. (d) is impossible by Theorem 10 .

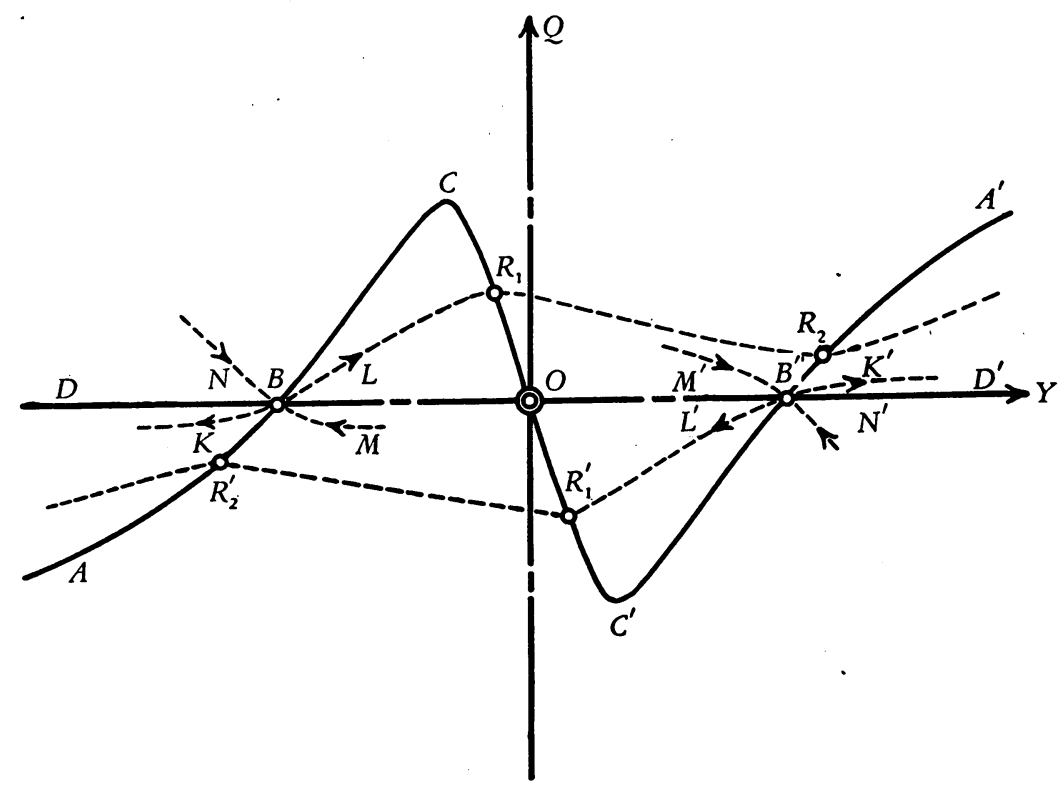

FIG. 2.

The theorem is accordingly proved, provided we can rule out the possibility (e) pictured in Figure 2 by $B^{\prime} L^{\prime} R_{1}^{\prime} R_{2}^{\prime}$. Paired with a motion on this arc, there is a motion symmetric to it with respect to $O$ on the $\operatorname{arc} B L R_{1} R_{2}$. These two arcs taken with $B^{\prime} R_{2}, B R_{2}^{\prime}$ bound a region containing $O$ and the motion $M^{\prime} B^{\prime}$ in its entirety. The motion $M^{\prime} B^{\prime}$ would possess $O$ or $B$ as $\alpha$-limiting point and $B^{\prime}$ as $\omega$-limiting point, thus contradicting Theorem 10 . Hence (e) is impossible.

\section{ThE RESTRICTED PROBLEMS OF ELLIPTIC AND HYPERBOLIC TYPE}

1. The differential equations of motion. If the mass $\mu$ moves in an elliptic 
[hyperbolic] orbit about the mass $1-\mu$, it is known $\left({ }^{19}\right)$ that

$$
\begin{array}{ll}
\rho=a(1-e \cos \phi), & a^{3 / 2}(\phi-e \sin \phi)=\kappa \tau, \\
{[\rho=a(e \cosh \psi-1),} & \left.a^{3 / 2}(e \sinh \psi-\psi)=\kappa \tau\right] .
\end{array}
$$

The independent variable $\bar{t}$ in IV turns out to be proportional to $\phi[\psi]$ and we find

so that

$$
\rho=a\left(1-e \cos \bar{t} / a^{\dot{1} / 2}\right), \quad\left[\rho=a\left(e \cosh \left(\bar{t} / a^{1 / 2}\right)-1\right)\right],
$$

$$
\begin{array}{rlrl}
l & =\log a\left(1-e \cos \bar{t} / a^{1 / 2}\right), & & {\left[l=\log a\left(e \cosh \left(\bar{t} / a^{1 / 2}\right)-1\right)\right],} \\
k & =\frac{e \sin \bar{t} / a^{1 / 2}}{a^{1 / 2}\left(1-e \cos \bar{t} / a^{1 / 2}\right)}, & {\left[k=\frac{e \sinh \bar{t} / a^{1 / 2}}{a^{1 / 2}\left(e \cosh \left(\bar{t} / a^{1 / 2}\right)-1\right)}\right],}
\end{array}
$$

are to be taken in the canonical equations (25) with $H$ defined as in (18) and the dot denoting differentiation with respect to $\bar{t}$.

For rectilinear problems the independent variable $t$ in IV is employed to yield

$$
\begin{array}{llrl}
\rho & =2 a \operatorname{sech}^{2} t / 2^{1 / 2}, & & {\left[\rho=2 a \operatorname{csch}^{2} t / 2^{1 / 2}\right],} \\
k & =-\left(1 / 2^{1 / 2}\right) \tanh t / 2^{1 / 2}, & & {\left[k=-\left(1 / 2^{1 / 2}\right) \operatorname{coth} t / 2^{1 / 2}\right] .}
\end{array}
$$

The differential equations (1) take the simple form

$$
\ddot{x}+k \dot{x}=\Omega_{x}, \quad \ddot{y}+k \dot{y}=\Omega_{y} .
$$

It is readily verified that the differential equations for the two types interchange when $t$ is replaced by $t+\left(\pi / 2^{1 / 2}\right) i$. One obtains the canonical form (25) for these equations by taking the above values of $k$ and placing $b=0$ in the definition of $H$ in (16).

2. Limiting motions. In the general problem of hyperbolic type $e>1$ and the $\omega$-limiting motions are the motions

$$
\begin{aligned}
x & =x_{0}+a^{1 / 2} p_{0}\left(1-e^{-\bar{t} / a^{1 / 2}}\right), & y & =y_{0}+g_{0} a^{1 / 2}\left(1-e^{-i / a 1 / 2}\right), \\
p & =p_{0} e^{-\bar{t}_{/} a^{1 / 2}}, & q & =q_{0} e^{-\bar{t} / a^{1 / 2}} .
\end{aligned}
$$

The $\omega$-limiting motions of a positively stable motion are equilibrium motions in (30). However since these equilibrium motions do not form a discrete set, it cannot be assumed that the motion tends uniformly towards a definite equilibrium motion, for it is conceivable that the motion tends toward a set of equilibrium motions.

For rectilinear problems of elliptic or hyperbolic type, it follows from (29) that the $\alpha$-and $\omega$-limiting motions are, respectively, motions of the dissipative and acquisitive rectilinear problems of parabolic type. A positively [negatively] stable motion approaches one of the equilibrium motions $E_{i}$ of the rectilinear problem of parabolic type uniformly as $t \rightarrow+\infty[-\infty]$. It will be observed that as $t \rightarrow \pm \infty$ the finite masses tend towards collision.

(19) See F. R. Moulton, op. cit., pp. 158-159 and pp. 177-178.

UNIVERSITY OF MARYLAND, College Park, Md. 